\title{
CLASSIFICATION OF LiDAR DATA WITH POINT BASED CLASSIFICATION METHODS
}

\author{
N. Yastikli and Z. Cetin \\ YTU, Civil Engineering Faculty, Department of Geomatics Engineering, 34210 Davutpasa, Istanbul, Turkey - \\ (ynaci, zerisir)@yildiz.edu.tr
}

Commission III, WG III/2

KEY WORDS: LiDAR, Point Cloud, Classification, Point Based, Vegetation, Building, Ground

\begin{abstract}
:
LiDAR is one of the most effective systems for 3 dimensional (3D) data collection in wide areas. Nowadays, airborne LiDAR data is used frequently in various applications such as object extraction, 3D modelling, change detection and revision of maps with increasing point density and accuracy. The classification of the LiDAR points is the first step of LiDAR data processing chain and should be handled in proper way since the $3 \mathrm{D}$ city modelling, building extraction, DEM generation, etc. applications directly use the classified point clouds. The different classification methods can be seen in recent researches and most of researches work with the gridded LiDAR point cloud. In grid based data processing of the LiDAR data, the characteristic point loss in the LiDAR point cloud especially vegetation and buildings or losing height accuracy during the interpolation stage are inevitable. In this case, the possible solution is the use of the raw point cloud data for classification to avoid data and accuracy loss in gridding process. In this study, the point based classification possibilities of the LiDAR point cloud is investigated to obtain more accurate classes. The automatic point based approaches, which are based on hierarchical rules, have been proposed to achieve ground, building and vegetation classes using the raw LiDAR point cloud data. In proposed approaches, every single LiDAR point is analyzed according to their features such as height, multi-return, etc. then automatically assigned to the class which they belong to. The use of un-gridded point cloud in proposed point based classification process helped the determination of more realistic rule sets. The detailed parameter analyses have been performed to obtain the most appropriate parameters in the rule sets to achieve accurate classes. The hierarchical rule sets were created for proposed Approach 1 (using selected spatial-based and echo-based features) and Approach 2 (using only selected spatialbased features) and have been tested in the study area in Zekeriyaköy, Istanbul which includes the partly open areas, forest areas and many types of the buildings. The data set used in this research obtained from Istanbul Metropolitan Municipality which was collected with 'Riegl LSM-Q680i' full-waveform laser scanner with the density of 16 points $/ \mathrm{m}^{2}$. The proposed automatic point based Approach 1 and Approach 2 classifications successfully produced the ground, building and vegetation classes which were very similar although different features were used.
\end{abstract}

\section{INTRODUCTION}

The airborne laser scanners (ALS) in other word LiDAR (Light Detection and Ranging) is directly measures 3D coordinates of objects and obtained results are the dense point clouds (Vosselman, 2009). The point clouds including the points on terrain (ground), vegetation, building and etc. which belong to the terrain and off-terrain objects are recorded during the laser scanning (Hao et al., 2009). The LiDAR point clouds are mostly used in object extraction, Digital Terrain Model (DTM) generation, 3D building modelling and change detection applications (Vosselman, 2000; Rottensteiner, 2003; Brenner, 2005; Hommel, 2009; Champion et al., 2009). The LiDAR point classification is the first step of LiDAR data processing in 3D city modelling, building extraction, DEM generation applications. In this LiDAR point classification step, each LiDAR point is classified into the meaningful categories such as ground, vegetation and building based on the LiDAR data properties. The accurate classification is crucial to achieve accurate 3D city models, building extraction, DEM generation because the result of the classification is directly used in these applications (Charaniya et al., 2004).

The techniques used in LiDAR point classification require the regular distributed (gridded) data or irregular distributed raw LiDAR data. The gridded LiDAR is mostly preferred data structure because of the potential use of the image processing methods (Clode et al., 2004). The characteristic point loss especially on vegetation and buildings, and losing height accuracy (in the interpolation stage of gridding process) are the disadvantages of using the gridded LiDAR data (Antonarakis et al., 2008). To eliminate characteristic point loss and losing height accuracy, most of the proposed classification algorithms are focus on classification of the irregular distributed raw LiDAR data. In raw LiDAR data classification, each 3D irregular distributed point is assigned to a semantic object class in the classification stage using LiDAR data properties such as the multi-returns, elevations, intensity, scan angle, etc. (Niemeyer et al., 2014). Since the manual processing is costly and time-consuming, the automation of classification task is highly needed (Moussa and El-Sheimy, 2010). The recent researches in the classification of the LiDAR data are focus on developing effective classification techniques for raw data to avoid time-consuming interactive editing (Forlani et al., 2006).

The aims of this study is to propose approaches for automatic point based classification of raw LiDAR point cloud to eliminate the problems experienced in working with gridded LiDAR data. The hierarchical rules using the raw LiDAR point features were generated in Approach 1 and Approach 2 to assign LiDAR points to proper ground, vegetation and building classes. 
Considering this aims, the paper is organized as follows: the next section describes the methodologies which includes the details of proposed automatic point based classification approaches. This is followed by the study area and data set. The experimental section provides the detailed information about the automatic point based classification of raw LiDAR point cloud with proposed Approach 1 and Approach 2. The concluding remarks are given in last section.

\section{METHODOLOGY}

The each LiDAR points should be assigned to the appropriate object class during the point based classification stage. The LiDAR point features obtained or calculated from 3D LiDAR point cloud are used in automatic point based classification (Mallet et al., 2011; Kim and Sohn, 2013). These features used in point based classification are identified in accordance with the targeted classes and the appropriate parameters of the features usually determined as a result of training stages. The used features in point based classification algorithms can be grouped as spatial-based features, echo-based features and waveform-based features (Mallet et al., 2011). The spatial-based and echo-based features can be computed from traditional multiple-pulse data. Waveform-based features are specifically used with full-waveform LiDAR data. The local environment, height features, eigenvalue features, local plane features, plane slope, homogeneity of surface normal, surface roughness, convex hull-based features, vertical profile, point density and density ratio are the some of the LiDAR point cloud's spatial features used in point based classification (Mallet et al., 2011). Echo-based features such as terrain echo, vegetation echo, power-line echo and building echo are determined using the pulses return from the surface. The laser pulses combined with the number of points corresponding to single, first, intermediate and last return can be used also determination of the echo-based features (Kim and Sohn, 2010). The echo amplitude, echo width, echo shape and echo cross-section are the waveformbased features used in point based classification (Chehata et al., 2009).

In this study, approaches (Approach 1 and Approach 2) have been proposed for the automatic point based classification of raw LiDAR point cloud with the combine use of spatial-based and echo-based features of LiDAR point cloud to eliminate the problems experienced in working with gridded LiDAR data. The spatial-based and echo-based features are used together in Approach 1. In Approach 2, only spatial-based features are used for automatic point based classification. The hierarchical rule sets were created for proposed Approach 1 and Approach 2 for automatic point based classification of raw LiDAR point cloud with defined routines. Figure 1 shows the workflow of the proposed automatic point based classification strategy with raw LiDAR point cloud. In order to prevent misclassification, rule sets were developed for automatic building extraction.

The determination of appropriate parameter for the selected features used in the routines is the important step for success of the automatic point based classification. The detailed parameter analyses have been performed to acquire most appropriate parameters used in Approach 1 and Approach 2 rule sets. The parameter analyses for ground and vegetation were conducted using the same pilot area. A different pilot area was used to determine appropriate building class parameters. In Figure 2, vegetation class parameter analyses before and after was given in the pilot area. Figure 3 shows the building class parameter analyses before and after in building pilot area. The same parameter sets determined in parameter analyses stage were used in Approach 1 and Approach 2 to eliminate the effect of the different parameter sets on classification results. The hierarchical rule sets for proposed Approach 1 and Approach 2 were created in TerraScan module of TerraSolid software.

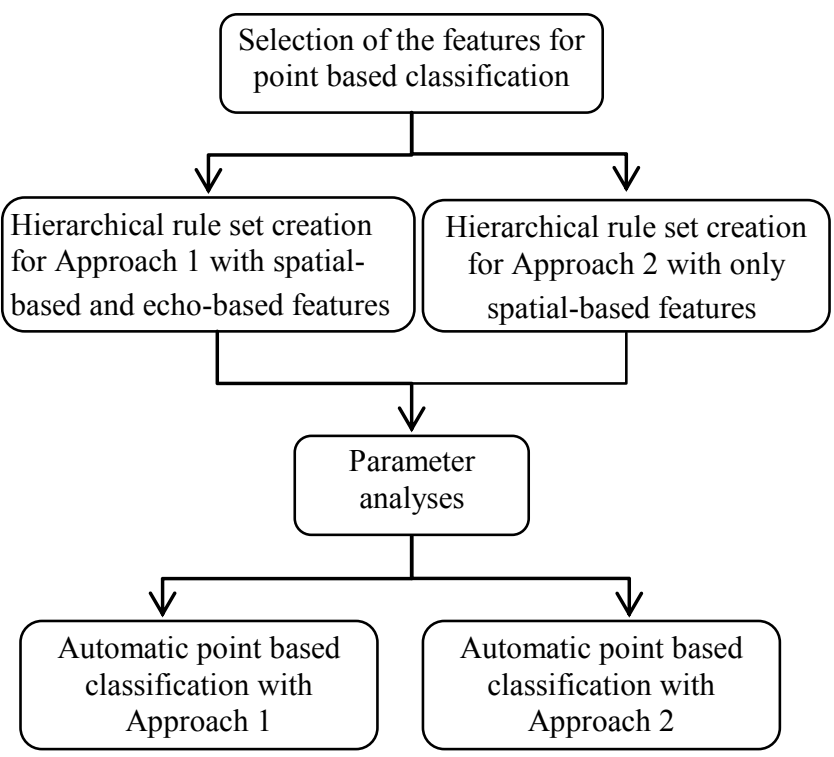

Figure 1. Workflow of the proposed automatic point based classification strategy with Approach 1 and Approach 2.
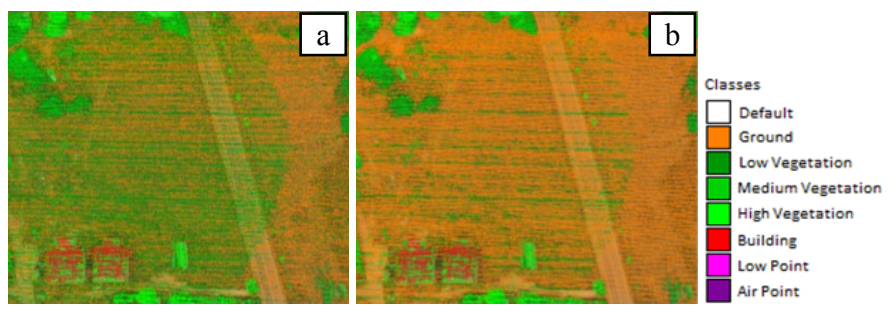

Figure 2. The results of the vegetation class parameter analyses before (a) and after (b).
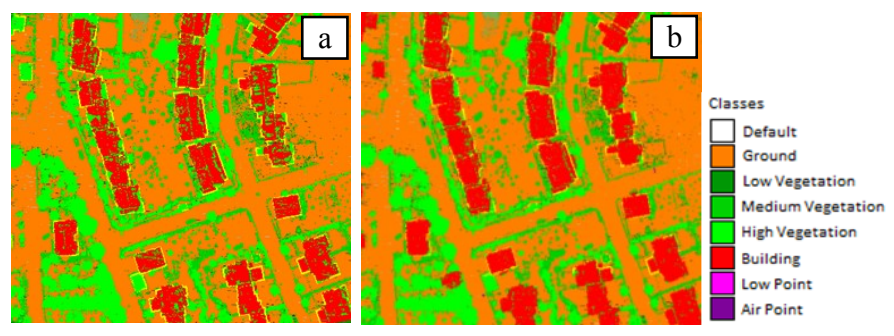

Figure 3. The results of the building class parameter analyses before (a) and after (b).

\section{STUDY AREA AND DATA SET}

The study area is located in Zekeriyakoy, Istanbul, Turkey has partly open areas, forest areas and many types of the buildings for the automatic point based classification of raw LiDAR point cloud with proposed Approach 1 and Approach 2 (Figure 4). The LiDAR data was collected with 'Riegl LSM-Q680i' fullwaveform laser scanner mounted 'Eurocopter AS350' in September, 2013 as a part of 'Digital Surface Model and 3D City Models Production with Air LiDAR Technology Project'. 
Figure 5 shows the LiDAR point cloud of the test area with the density of 16 points $/ \mathrm{m}^{2}$. Some technical properties of the 'Riegl LSM-Q680i' full-waveform laser scanner is given in Table 1. The obtained point clouds from Istanbul Metropolitan Municipality was in LAS (Log ASCII Standard) format and classified with standard parameters including the ground, low vegetation, medium vegetation, high vegetation, building, low point and air point classes.

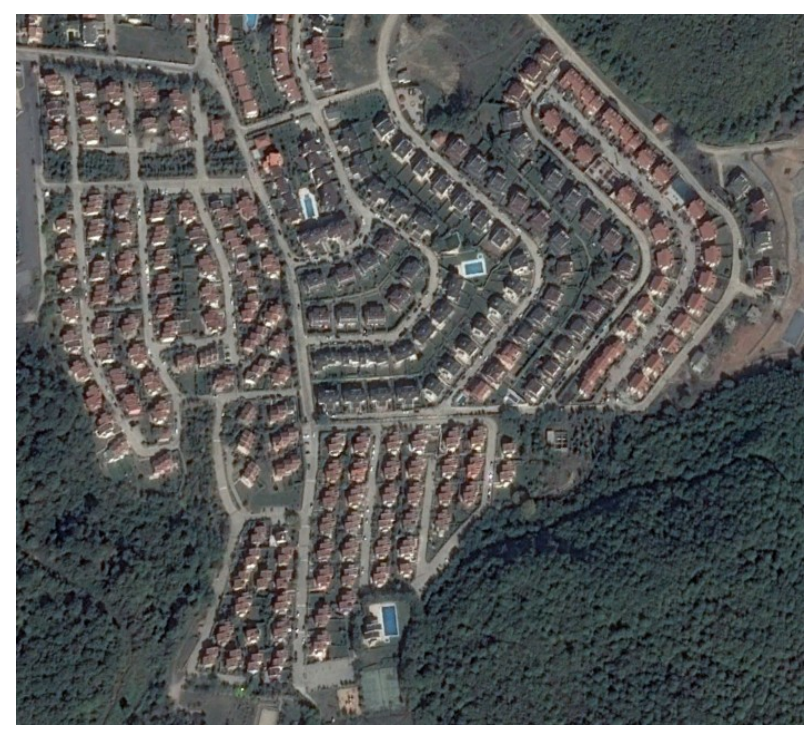

Figure 4. Study area in Istanbul.

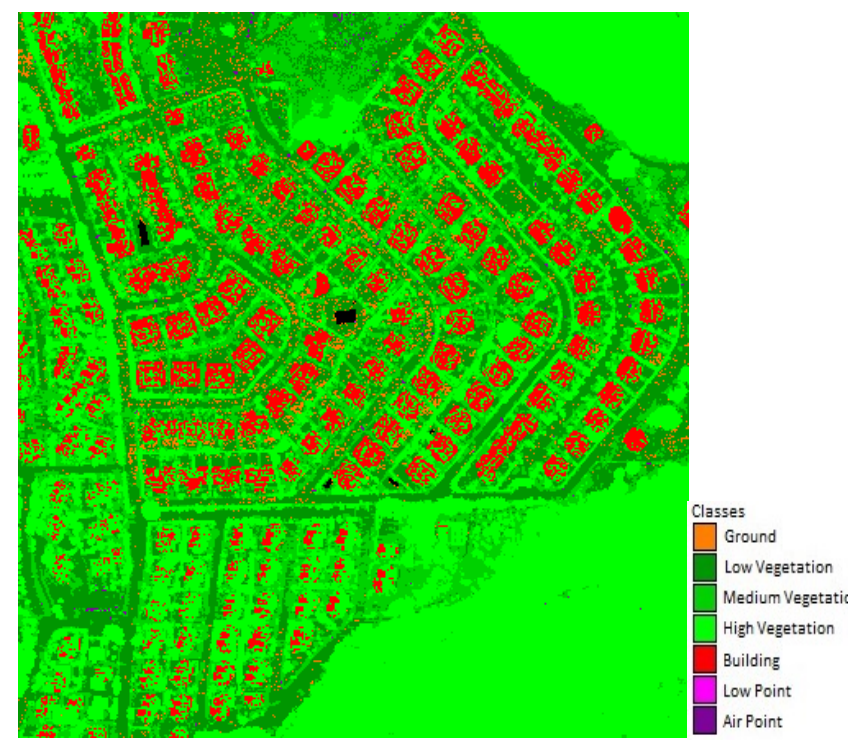

Figure 5. The roughly classified points cloud of the test area with standard parameters.

\section{EXPERIMENT}

The automatic point based classifications with proposed Approach 1 and Approach 2 have been achieved using the LiDAR point cloud of study area. As a result of the automatic point based classification with Approach 1 and Approach 2, the ground, low vegetation, medium vegetation, high vegetation, building, default, low point and air point classes were obtained. The low, medium and high vegetation classes were combined into a single vegetation class for both Approach 1 and Approach 2 since only ground, vegetation and building classes was aimed. With the same purposes, default, low point and air point classes were combined into a single class named as other. The results of the automatic point based classification with proposed Approach 1 (with spatial-based and echo-based features) and Approach 2 (with spatial-based features) were given in Figure 6 and Figure 7 respectively using the created hierarchical rule sets in TerraScan module of TerraSolid software.

\begin{tabular}{|l|l|}
\hline Laser pulse repetition rate & up to $400000 \mathrm{~Hz}$ \\
\hline Effective measurement rate & $\begin{array}{l}\text { up to } 266 \mathrm{kHz} @ 60^{\circ} \mathrm{scan} \\
\text { angle }\end{array}$ \\
\hline Laser wavelength & near infrared \\
\hline Laser beam divergence & $\leq 0.5 \mathrm{mrad}$ \\
\hline Scanning mechanism & rotating poligon mirror \\
\hline Scan pattern & parallel scan lines \\
\hline Scan angle range & $+/-30^{\circ}=60^{\circ}$ total \\
\hline Scan speed & $10-200$ lines $/ \mathrm{sec}$ \\
\hline Angle measurement resolution & $0.001^{\circ}$ \\
\hline Intensity Measurement & $\begin{array}{l}\text { high resolution 16-bit } \\
\text { intensity information }\end{array}$ \\
\hline
\end{tabular}

Table 1. Technical information of 'Riegl LSM-Q680i' laser scanner.

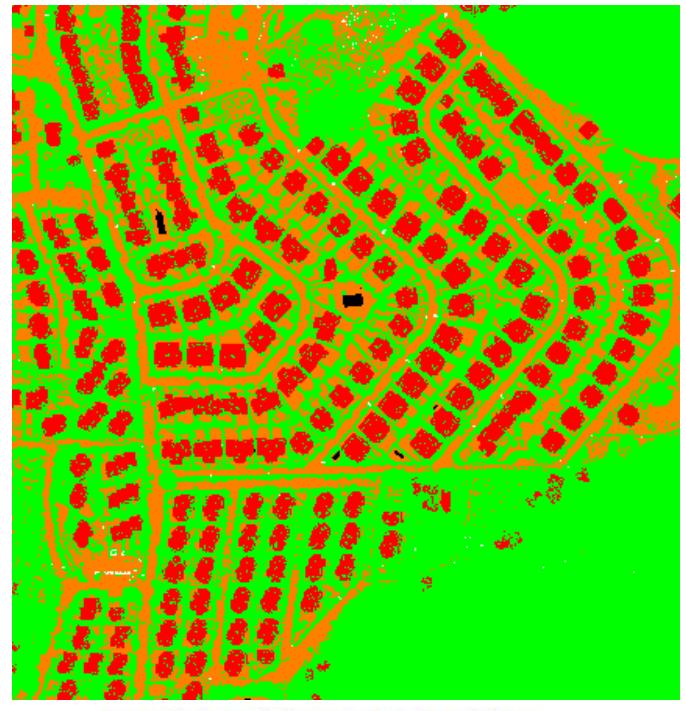

$$
\text { Classes } \mid \square \text { Ground } \square \text { vegetation } \square \text { Building } \square \text { other }
$$

Figure 6. The classified points cloud of the test area with Approach 1 (with spatial-based and echo-based features).

The automatic point based classification results with Approach 1 (see Figure 6) and Approach 2 (see Figure 7) produced accurate ground classes against with the roughly classified point cloud (see Figure 5). Although the Approach 1 used both spatial-based and echo-based features and Approach 2 used only spatial-based features, the results of the automatic point based classification were almost same. The ground and building points incorrectly assigned to the vegetation classes in the roughly classified points cloud were successfully assigned to the correct ground and building classes with the proposed Approach 1 and Approach 2 (see Figure 5, Figure 6 and Figure 7). The obtained automatic point based classification results in the study area with proposed Approach 1 and Approach 2 approved the success of the methodology. 


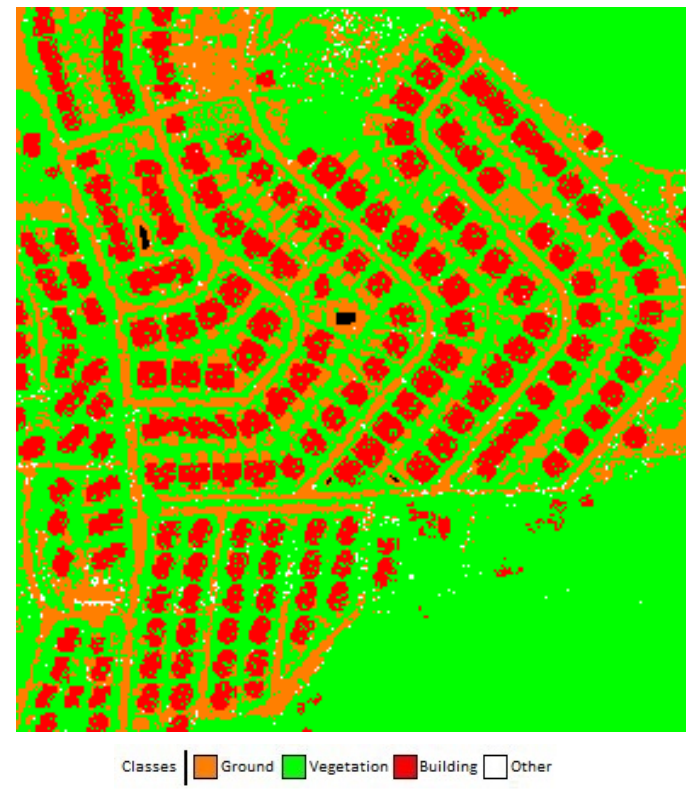

Figure 7. The classified points cloud of the test area with Approach 2 (with spatial-based features).

\section{CONCLUSIONS}

In this study, efficient workflow for the automatic point based classification of LiDAR data was presented and the hierarchical rules have been developed for proposed Approach 1 and Approach 2. In Approach 1, both spatial-based and echo-based features were used together. In Approach 2, only spatial-based features were used for the automatic classification. The proposed approaches have been tested in the study area which has the partly open areas, forest areas and many types of the buildings in Zekeriyakoy, Istanbul, Turkey. To determine most appropriate parameters in the rule sets, the detailed parameter analyses have been performed in the pilot areas using the LiDAR data of the study area. The same parameter sets determined by parameter analyses in the pilot areas were used with the Approach 1 and Approach 2. As can be seen in Figure 5, Figure 6 and Figure 7, most of the misclassified ground, vegetation and building points in roughly classification results have been correctly assigned in proposed the automatic point based classification results with Approach 1 and Approach 2 . The automatic point based classification results were almost same for proposed approaches, although the Approach 1 use both the spatial-based and echo-based features and Approach 2 use only the spatial-based features.

The obtained results of this research verified that the automatic point based classification of raw LiDAR point can be successfully performed with proposed Approach 1 and Approach 2. The characteristic point loss and losing height accuracy in gridded LiDAR data can be eliminated with proposed automatic point based classification approaches. The results of the automatic point based classification can be used successfully for generation of the more realistic 3D building model, urban and telecommunication planning, disaster management real-time simulations for training and other applications with $3 \mathrm{D}$ city models.

\section{ACKNOWLEDGEMENTS}

We would like to thank the Metropolitan Municipality of Istanbul for the LiDAR data set of the test area.

\section{REFERENCES}

Antonarakis, A. S., Richards, K. S., Brasington, J., 2008. Object-Based Land Cover Classification Using Airborne LiDAR. Remote Sensing of Environment, 112(6), pp. 29882998.

Brenner, C., 2005. Building Reconstruction From Images and Laser Scanning. International Journal of Applied Earth Observation and Geoinformation, 6(2005), pp. 187-198.

Champion, N., Rottensteiner, F., Matikainen, L., Liang, X., Hyyppä, J., Olsen, B. P., 2009. A Test of Automatic Building Change Detection Approaches. In: The International Archives of the Photogrammetry, Remote Sensing and Spatial Information Sciences, Paris, France, Vol. XXXVIII, Part 3/W4, pp. $145-150$.

Charaniya, A. P., Manduchi, R., Lodha, S. K., 2004. Supervised Parametric Classification of Aerial LiDAR Data. In: Computer Vision and Pattern Recognition Workshop, 2004, Washington, DC, USA, Vol. 3, pp. 30-30.

Chehata, N., Guo, L., Mallet, C., 2009. Airborne LiDAR Feature Selection For Urban Classification Using Random Forests. In: The International Archives of the Photogrammetry, Remote Sensing and Spatial Information Sciences, Paris, France, Vol. XXXVIII, Part 3/W8, pp. 207-212.

Clode, S. P., Kootsookos, P., Rottensteiner, F., 2004. The Automatic Extraction of Roads From Lidar Data. In: The International Archives of Photogrammetry, Remote Sensing and Spatial Information Sciences, Istanbul, Turkey Vol. XXXV, Part B3, pp. 231-236.

Forlani, G., Nardinocchi, C., Scaioni, M., Zingaretti, P., 2006. Complete Classification of Raw LiDAR Data and 3D Reconstruction of Buildings. Pattern Analysis \& Applications, 8(4), pp.357-374.

Hao, Z., Zhang, Y., Liu, J., Ji, S., 2009. Automatic Building Detection Using Airborne LiDAR Data. In: International Forum on Information Technology and Applications, Vol. 3, pp. 668-671.

Hommel, M., 2009. Verification of A Building Damage Analyses and Extension To Surroundings of Reference Buildings. In: The International Archives of the Photogrammetry, Remote Sensing and Spatial Information Sciences, Paris, France, Vol. XXXVIII, Part 3-W8, pp. 18- 23.

Kim, H. B., Sohn, G., 2010. 3D Classification of Power-Line Scene From Airborne Laser Scanning Data Using Random Forests. In: The International Archives of Photogrammetry, Remote Sensing and Spatial Information Sciences, Paris, France, Vol. XXXVIII, Part 3A, pp. 126-132.

Kim, H. B., Sohn, G., 2013. Point-Based Classification of Power Line Corridor Scene Using Random Forests. Photogrammetric Engineering and Remote Sensing, 79(9), pp 821-833.

Mallet, C., Bretar, F., Roux, M., Soergel, U., Heipke, C., 2011. Relevance Assessment of Full-Waveform Lidar Data For Urban 
Area Classification. ISPRS Journal of Photogrammetry and Remote Sensing, 66(6), pp. 71-S84.

Moussa, A., El-Sheimy, N., 2010. Automatic Classification and 3D Modeling of Lidar Data. In: The International Archives of Photogrammetry, Remote Sensing and Spatial Information Sciences, Paris, France, Vol. XXXVIII, Part 3B, pp. 155-159.

Niemeyer, J., Rottensteiner, F., Soergel, U., 2014. Contextual Classification of LiDAR Data and Building Object Detection In Urban Areas. ISPRS Journal of Photogrammetry and Remote Sensing, 87(2014), pp. 152-165.

Rottensteiner, F., 2003. Automatic Generation of High-Quality Building Models From LiDAR Data. IEEE Computer Graphics and Applications, 23(6), pp. 42-50.

Vosselman, G., 2000. Slope Based Filtering of Laser Altimetry Data. In: The International Archives of the Photogrammetry, Remote Sensing and Spatial Information Sciences, Amsterdam, The Netherlands, Vol. XXXIII, Part B3, pp. 935-942.

Vosselman, G., 2009. Advanced Point Cloud Processing. In: $D$. Fritsch (Ed.), Photogrammetric Week 2009, Heidelberg, Germany, pp. 137-146. 